\title{
La Fraternité réveillée
}

Jordi Riba et Patrice Vermeren (sous la direction de), 2014, L'Harmattan, $213 \mathrm{p}$.

\section{Abdoulaye Elimane Kane}

\section{OpenEdition}

\section{Journals}

Édition électronique

URL : https://journals.openedition.org/ries/6035

DOI : $10.4000 /$ ries. 6035

ISSN : 2261-4265

Éditeur

France Education international

\section{Édition imprimée}

Date de publication : 1 décembre 2017

Pagination : $32-33$

ISBN : 978-2-85420-616-6

ISSN : 1254-4590

Référence électronique

Abdoulaye Elimane Kane, "La Fraternité réveillée », Revue internationale d'éducation de Sèvres [En ligne], 76 | décembre 2017, mis en ligne le 01 décembre 2017, consulté le 25 juin 2021. URL : http://

journals.openedition.org/ries/6035; DOI : https://doi.org/10.4000/ries.6035

Ce document a été généré automatiquement le 25 juin 2021.

(c) Tous droits réservés 


\title{
La Fraternité réveillée
}

Jordi Riba et Patrice Vermeren (sous la direction de), 2014, L'Harmattan, $213 \mathrm{p}$.

\author{
Abdoulaye Elimane Kane
}

\section{RÉFÉRENCE}

La Fraternité réveillée, Jordi Riba et Patrice Vermeren (sous la direction de), 2014,

L'Harmattan, $213 \mathrm{p}$.

$1 \quad$ La Fraternité réveillée est un ouvrage collectif composé de treize textes dus à: Sophie Wahnich, Robert Damien, Georges Navet, Pierre Crétois, Laurence Cornu, Jordi Riba, Patrice Vermeren, Angel Puyol, Laura Llevadot, Geneviève Fraisse, Bérengère Kolly et Marc Thomassin. Il comprend des contributions nouvelles et des commentaires notamment sur les thèses de Saint-Just, J.-P. Sartre, Pierre Leroux, Miguel Abensour, Derrida.

\section{Quatre entrées pour une lecture transversale de cet ouvrage}

2 Si le constat unanime est fait de l'existence d'une racine chrétienne de la notion de fraternité, celle-ci est vite relativisée au profit de conceptions laïques, sociales et politiques. Nos auteurs la rattachent volontiers à la Révolution française et à la devise républicaine. Certains déplorent la relégation de ce principe à un rang subalterne par rapport à ceux de liberté et d'égalité, voire sa réduction à une sorte de "pensée molle ", tout juste bonne aux invocations lyriques. D'autres, au contraire, lient sa place dans la devise républicaine et sa portée révolutionnaire à une dimension sacrée : «la fraternité ou la mort » (Saint-Just) ; « fraternité d'Apocalypse » (Sartre) ; « un événement de haute température noué aux émotions du danger encouru en commun et d'un sauvetage en commun » (Sartre). 
3 L'analyse étymologique révèle aussi le caractère polysémique du terme fraternité : signification biologique et familiale qui fonde sur les liens de sang l'idée d'union sacrée ; fictions, interprétations, transpositions, extrapolations et multiples usages $d u$ terme.

4 Dans le même registre, à des degrés divers, une interrogation récurrente traverse l'ouvrage: la notion de fraternité est-elle interchangeable avec celles de solidarité, d'égalité et d'amitié?

5 À ceux qui considèrent que la fraternité ne peut se concevoir sans l'égalité et la liberté - les deux autres principes de la devise républicaine - répondent ceux qui exigent l'identification de ses conditions spécifiques de possibilité. Cependant, tous s'accordent sur ceci : la fraternité ne peut reposer ni sur l'intérêt personnel ni sur la recherche de gain et elle récuse tout motif utilitariste. Soucieux de réveiller (et de révéler) les valeurs de cette fraternité, certains auteurs soulignent les limites de ce concept et en proposent d'autres.

\section{Alternatives au concept de fraternité}

6 Premier indice de la fragilité de la notion de fraternité : le paradoxe des fratricides. Les inimitiés tragiques entre frères devraient être impossibles, inconcevables. Les mythes fraternels confirment leur existence et rappellent qu'ils ont parfois été fondateurs d'ordres nouveaux. Parmi les exemples emblématiques: Abel et Caïn; Romus et Romulus.

7 Deuxième indice : le caractère discriminatoire de la notion de fraternité. Son contenu sémantique et sa fonction sociale participent, selon certains auteurs, de la production et/ou la reproduction d'un ordre social inégalitaire. Exclusion des sœurs, cousines, mères, nièces, etc., au niveau familial. Idéologie fondée sur le schème phallocratique de subordination de la femme à l'homme.

8 Pour pallier les insuffisances, ambiguïtés ou paradoxes du terme "fraternité ", des concepts de substitution affublés de desseins explicitement politiques et/ou idéologiques sont proposés. Par exemple celui de filialité. D'origine chrétienne et familiale, comme la fraternité, cette notion est redéfinie au prix d'une subversion sémantique : pour rompre avec la "projection narcissique » véhiculée par la paternité et la maternité, promouvoir le statut de «fils adoptif », symbole de " la transcendance désirée que l'on aime au-delà de tout calcul et de toute réciprocité ». Condition essentielle pour "un nouvel horizon à l'éthique du politique» (L. Llevadot). Et l'avènement d'une démocratie ouverte.

9 Autre concept de substitution: la fraternisation. Postulat: il n'existe ni fraternité naturelle ni fraternité raciale; et sa réalisation universelle demeure un horizon lointain. Alternative : restreindre le champ de cette visée humaniste à des pratiques classables sous le vocable de fraternisation, expériences "d'adhésion volontariste et volontaire des frères entre eux ». Derrida la définit ainsi :

C'est ce qui produit symboliquement, conventionnellement, par engagement assermenté, une politique déterminée.

10 Il y a enfin une manière de synthèse dans une contribution portant sur les « figures de la fraternité ». Les intitulés de la typologie que propose L. Cornu sont significatifs : «expérience initiatique, "égale condition»; «fraternisations»; «fraternités et 
puissances d'agir en commun »; "fiabilité et solidarité »; «fidélité oblige »; « réel, imaginaire, symbolique », « tous uns ».

11 Galerie significative de portraits et question ultime qui, en fait, traverse tout le livre : au service de quoi sont toutes ces figures de la fraternité ? Réponse : l'acceptation et la recherche du commun. Non pas sous les espèces d'un culte mais par construction, culture et entretien de la fraternité. Dans des lieux non pas sacrés mais communs, caractérisés par «des immanences sensibles", valeurs de la communauté politique et de la république.

12 Voici donc treize contributions révélatrices de la possibilité de (re)penser l'humain par le biais du concept de fraternité - courant et problématique - soumis à une lecture ouverte de l'histoire concrète des hommes.

\section{AUTEUR}

\section{ABDOULAYE ELIMANE KANE}

Abdoulaye Elimane Kane est professeur émérite de philosophie (Université Cheikh Anta Diop, Dakar). Son doctorat d'État (Lille, 1987) portait sur les systèmes de numération parlée des langues ouest-africaines (Lille, 1987). Il a exercé plusieurs responsabilités, en tant qu'inspecteur général de philosophie, chef du département de philosophie (1987-89), ministre de la communication et de la culture du Sénégal (1993-2000). Il est également romancier. Courriel : elkane31@hotmail.fr 